\title{
Prevalência do consumo de substâncias psicoativas entre estudantes de medicina no interior do Nordeste brasileiro
}

\section{Prevalence of consumption of psychoactive substances among medicine students in the interior of Northeastern Brazil}

\author{
Sabrina Mércia dos Santos Siebra' (1) | sabrinasiebra@alu.uern.br \\ Tassio Danilo Rego de Queiroz' (1) tassioqueiroz@alu.uern.br \\ Eudes Euler de Souza Lucena² (D) eudeseuler@hotmail.com \\ Allyssandra Maria Lima Rodrigues Maia' (D) allyssandramr@hotmail.com \\ Ubirajara Caldas Leonardo Nogueira Junior' 1 (D) ubiracaldas.md@gmail.com \\ Álvaro Marcos Pereira Lima' (1) alvarolima23@yahoo.com.br
}

\section{RESUMO}

Introdução: No contexto da formação em Medicina, muitos estudantes buscam alívio e equilíbrio emocional por meio do uso de substâncias psicoativas.

Objetivo: Este estudo teve como objetivo avaliar o consumo de substâncias psicoativas entre os acadêmicos de Medicina da Faculdade de Ciências da Saúde da Universidade do Estado do Rio Grande do Norte (UERN) com o intuito de contribuir para a formulação de atividades de prevenção.

Método: A pesquisa possui corte transversal e abordagem quantitativa e descritiva. Aplicaram-se questionários padronizados entre outubro e dezembro de 2020 para discentes do primeiro ano e do internato. Analisaram-se variáveis de natureza categórica por meio de frequências e percentuais, e a relação entre essas variáveis foi realizada pelo teste qui-quadrado ou exato de Fisher. As associações e comparações, em relação aos períodos, foram consideradas significativas no caso de $\mathrm{p}$-valor $<0,05$.

Resultado: A prevalência do uso de substâncias psicoativas na vida foi de $81,7 \%$ ( $n=107)$. O consumo de tabaco e Cannabis foi significativamente maior em mulheres em relação aos homens: $p=0,019$ e $p=0,05$, respectivamente. Além disso, 48,4\% dos discentes que têm insônia, $85,7 \%$ dos que relataram possuir dependência e $39 \%$ dos que acreditam que cursar Medicina é fator precipitante de consumo fazem uso de ansiolíticos, sedativos e hipnóticos: $p=0,025, p=0,004$ e $p=0,01$, respectivamente.

Conclusão: Observaram-se um elevado uso de substâncias psicoativas entre os estudantes de Medicina da UERN e a manutenção do perfil de uso ao longo dos períodos do curso com a presença de achados atípicos que divergiram de outros trabalhos, evidenciando a heterogeneidade das populações estudantis médicas, o que aponta para a necessidade de mais estudos para ampliação e análise dos resultados encontrados.

Palavras-chave: Abuso Oral de Substâncias; Estudantes de Medicina; Qualidade de Vida.

\section{ABSTRACT}

Introduction: In the context of medical training, many students seek relief and emotional balance through the use of psychoactive substances.

Objective: This study aimed to evaluate the consumption of psychoactive substances among medical students at the Faculty of Health Sciences, State University of Rio Grande do Norte, with the aim of contributing to the formulation of prevention activities.

Method: This cross-sectional study adopted a quantitative and descriptive approach. Standardized questionnaires were applied between October and December 2020 for first-year and internship students. Categorical variables were analyzed using frequencies and percentages and the relationship between these variables was performed using the chi-square test or Fisher's exact test. Associations and comparisons, in relation to the study periods, were considered significant in the case of $p$-value $<0.05$.

Results: The lifetime prevalence of psychoactive substance use was $81.7 \%(n=107)$. Tobacco and cannabis consumption was significantly higher in women compared to men, $p=0.019$ and $p=0.05$, respectively. Furthermore, $48.4 \%$ of the students who suffer from insomnia, $85.7 \%$ of those who reported being substance-dependant, and 39\% of those who believe that studying medicine is a precursor for consumption, make use of anxiolytics, sedatives, and hypnotics, $p=0.025, p=0.004$ and $p=0.01$, respectively.

Conclusion: A high use of psychoactive substances was found among medical students at the State University of Rio Grande do Norte and the use profile maintained throughout the course included atypical findings that diverged from other studies, demonstrating the heterogeneity of medical student populations, which points to the need for more studies to expand and analyze the results found.

Keywords: Oral Substance Abuse; Medical Students; Quality of Life.

${ }^{1}$ Universidade do Estado do Rio Grande do Norte, Mossoró, Rio Grande do Norte, Brasil.

2 Universidade Federal do Rio Grande do Norte, Natal, Rio Grande do Norte, Brasil.

Editora-chefe: Rosiane Viana Zuza Diniz.

Editor associado: Roberto Zonato Esteves.

Recebido em 06/09/21; Aceito em 13/10/21.

Avaliado pelo processo de double blind review. 


\section{INTRODUÇÃO}

Determinadas substâncias, em razão de ocasionarem possíveis mudanças na funcionalidade neural central, tornaramse conhecidas como substâncias psicoativas (SPA). Esse grupo não inclui apenas drogas ilícitas, como maconha, cocaína e crack, mas também álcool, tabaco, benzodiazepínicos, entre outros. Tal ingestão, do ponto de vista da saúde pública, traz preocupação porque predispõe a alterações fisiológicas importantes, acidentes diversos, violências e comportamentos de risco, além de forte tendência a causar dependência de acordo com sua utilização'. Segundo dados apresentados no Relatório do United Nations Office on Drugs and Crime (UNODC) ${ }^{2}$, no ano de 2017, cerca de 217 milhões de pessoas entre 15 e 64 anos usaram alguma substância psicoativa pelo menos uma vez no ano anterior, o que correspondia a cerca de 5,5\% da população de todo o mundo nessa faixa etária, naquele ano.

$O$ ingresso do estudante no ambiente universitário consiste em múltiplos processos que envolvem aspectos externos, dos ambientes acadêmico e social, e aspectos internos do indivíduo, como a habilidade de encarar as diversas situações, as reações físicas psicossomáticas e os diferentes estados de humor $^{3}$. O contexto de formação em Medicina torna-se ainda mais denso, aglutinando responsabilidades sociais e técnicas desde a concorrência do vestibular, iniciando-se desde então uma dificuldade em estabelecer uma boa qualidade de vida 4 .

O primeiro ano da Faculdade de Medicina é um período de grandes expectativas, tendo em vista que muitos alunos têm as primeiras vivências no que diz respeito ao contato com a morte e a grande demanda de estudo. A carga horária extenuante, em turno integral, além da necessidade de novos hábitos de estudo, faz com o que o discente se prive de suas atividades de lazer, bem como abandone as práticas de atividade física ${ }^{5}$. Em contrapartida, nos dois últimos anos do estágio supervisionado obrigatório, também referido como internato, renovam-se as angústias. Esse é um momento marcado por considerável relevância, que praticamente obriga o discente a dedicar-se quase que exclusivamente à medicina.

Nesse contexto, muitos estudantes de Medicina buscam alívio e equilíbrio emocional por meio do uso de SPA ${ }^{6}$. Essas substâncias ativam o circuito neural de recompensa e prazer e são utilizadas pelos discentes com o intuito de buscar sensações prazerosas de bem-estar, permitindo, assim, o controle do estresse $^{7}$. Diversas pesquisas apontam para um consumo de drogas crescente no decorrer do curso médico, com o pico do consumo nos dois últimos anos, sendo as atividades práticas iniciadas no quinto ano do curso geradoras de ansiedade. Tal cenário soma-se com as exigências frequentes de frutos pelo seio familiar e pelo próprio discente, o que culmina, de forma direta, na ingestão desenfreada de certas drogas ${ }^{8}$. As pesquisas têm demonstrado índices elevados de consumo de drogas entre os acadêmicos desse curso, constituindo uma das inúmeras "válvulas de escape" para os problemas psicológicos ou de resiliência provocados pela rotina estressante?.

A Faculdade de Ciências da Saúde (FACS) da Universidade do Estado do Rio Grande do Norte (UERN), de acordo com seu projeto pedagógico, possui três ciclos: ensinos básico, profissionalizante (técnico/clínico) e prático (internato), com duração de dois anos cada. Esses ciclos utilizam-se de uma articulação entre as várias etapas do percurso educativo, o que implica uma sequencialidade progressiva, de modo a conferir a cada etapa as funções de completar, aprofundar e alargar a etapa anterior, numa perspectiva de continuidade e unidade global do ensino e aprendizagem, a fim de favorecer a transição adequada entre os ciclos. As disciplinas de farmacologia são lecionadas de acordo com a seguinte organização: farmacologia básica no quarto período, farmacologia aplicada I no quinto e farmacologia aplicada II no oitavo, sendo o conteúdo de dependência química e alcoolismo administrado nesta última. Logo, espera-se que, de acordo com o avanço dos semestres, os acadêmicos dos períodos finais do curso sejam mais responsáveis em relação ao consumo de SPA, tendo em vista que conhecem as consequências que elas podem causar a si próprios e à população ${ }^{10}$.

Lemos et al. ${ }^{11}$, em pesquisa realizada com 404 estudantes de Medicina das escolas médicas de Salvador, constataram elevados valores de frequência de uso de SPA durante a vida para álcool, lança-perfume, tabaco e maconha: 92,8\%, 46,2\%, $38,9 \%$ e $20,5 \%$, respectivamente. Nessa pesquisa, a diversão foi apontada como principal razão para o uso de SPA, com 58,7\%. A expectativa da comunidade é de que os profissionais da saúde, nesse caso os futuros médicos, sejam cientes dos efeitos nocivos dessas substâncias e as utilizem menos, quando comparados com a população no geral. Entretanto, isso não é visualizado na literatura nacional e internacional. Esse fato suscita a importância de as universidades oferecerem serviços de apoio psicológico, sobretudo quando existe dependência química.

Dessa forma, considerando a facilidade do acesso às SPA entre os estudantes universitários, a prevalência do uso abusivo e os potenciais riscos à saúde, torna-se relevante investigar esse contexto. Quando se analisou a produção científica sobre a temática, evidenciou-se uma lacuna no que tange aos estudos que abordam especificadamente estudantes de Medicina, sobretudo na Região Nordeste do Brasil. Sendo assim, esta pesquisa teve como objetivos analisar o consumo de SPA entre os acadêmicos de Medicina da FACS/UERN e verificar as substâncias mais utilizadas com o intuito de contribuir para a formulação de atividades de prevenção. Neste estudo, adotaram-se as seguintes variáveis: idade, sexo, cidade da origem, período do curso, insônia e estresse. 


\section{MÉTODO}

Efetuou-se um estudo transversal analítico com abordagem quantitativa. A pesquisa foi realizada com os estudantes de Medicina da UERN por meio da aplicação de questionários aos discentes do primeiro ano da faculdade e do internato, que compreende o quinto e sexto anos. A escolha dessa amostra deu-se em virtude de serem períodos extremos do curso, que compreendem alta carga de ansiedade e expectativas envolvidas. Dessa forma, pôde-se analisar e comparar se há diferenças no uso das SPA e, sobretudo, na frequência e consciência dos efeitos colaterais.

Realizou-se esse levantamento a partir de um questionário utilizando a ferramenta Google Forms e todos os convites para preenchimento foram enviados via WhatsApp. Os critérios de inclusão foram: ser aluno devida e regularmente matriculado na FACS/UERN no primeiro, quinto e sexto anos de curso e ter assinado o Termo de Consentimento Livre e Esclarecido (TCLE) que aparecia on-line. O questionário só começava após o respondente marcar "sim", informando que havia lido o TCLE e concordado com ele. Os critérios de exclusão foram: responder ao questionário de forma incompleta.

Para a coleta de dados, utilizou-se um questionário padronizado, de autopreenchimento e com questões objetivas. Esse questionário consistiu em uma adaptação do instrumento proposto e desenvolvido pela Organização Mundial da Saúde (OMS): Research and Reporting Project on the Epidemiology of Drug Dependence ${ }^{12}$. Um teste piloto foi realizado com cinco estudantes de Medicina que não foram incluídos na pesquisa.

O questionário foi composto por três seções. A primeira referia-se a dados sociodemográficos, incluindo sexo, idade, período do curso (primeiro ano ou internato) e se o estudante cursava Medicina na sua cidade de origem. A segunda interrogava aspectos sobre o uso de SPA: tabaco (cigarros, charutos, cigarrilhas etc.), álcool (cervejas, vinhos, licores, bebidas espirituosas, shots etc.), Cannabis (haxixe, erva, marijuana, pólen etc.), cocaína (coca, crack etc.), estimulantes do tipo anfetamina (speed, anfetaminas, ecstasy etc.), inalantes (cola, gasolina, óxido nitroso, solvente etc.), ansiolíticos, sedativos e hipnóticos, alucinógenos (dietilamida do ácido lisérgico (Lysergsäurediethylamid — LSD), cogumelos, fenciclidina (phenylcyclohexyl piperidine - PCP), ketamina etc.) e opiáceos (heroína, morfina, metadona, buprenorfina, codeína etc.). Abordaram-se ainda a frequência, a dependência e a relação com quadro de estresse e insônia. A terceira seção investigava as causas associadas a esse uso, a relação com a Faculdade de Medicina, as consequências e a consciência acerca dos efeitos do consumo.

O cálculo amostral foi realizado para estimar a proporção de alunos que fazem uso de SPA considerando uma amostragem aleatória simples. Consideraram-se um nível de confiança de 95\%, uma margem de erro de 5\% e uma proporção estimada de 0,5. Para uma população de 164 alunos, obteve-se um tamanho de amostra de 131, sendo 80 do internato e 51 do primeiro ano. Analisaram-se variáveis de natureza categórica por meio de frequências e percentuais, e a associação entre essas variáveis foi realizada pelo teste qui-quadrado ou exato de Fisher (no caso de valores esperados menores do que 5). Também se calculou o resíduo do teste qui-quadrado para verificar a significância da categoria. Associações e comparações, em relação aos períodos, foram consideradas significativas no caso de $p$-valor $<0,05$. Os dados foram tabulados em planilha do Microsoft Office Excel 2016 e analisados no programa Statistical Package for the Social Sciences (IBM SPSS Statistics 24).

O questionário foi aplicado entre outubro e dezembro de 2020, e todos os participantes assinaram o TCLE antes de responderem à pesquisa. O estudo foi aprovado pelo Comitê de Ética em Pesquisa da UERN, sob o no 4.295 .616 e Certificado de Apresentação de Apreciação Ética(CAAE) no 29323520.0.0000.5294.

\section{RESULTADOS}

No início do presente estudo, havia 59 alunos regularmente matriculados no primeiro ano e 104 no internato do curso de Medicina da UERN. A coleta de dados ocorreu entre outubro e dezembro de 2020, e obteve-se uma amostra de 51 discentes do primeiro ano (86\%) e 80 do internato (77\%), totalizando 131 respostas (80\%) do público-alvo. Consideraramse um nível de confiança de $95 \%$ e uma margem de erro de $5 \%$. O sexo masculino representou $60,3 \%(n=79)$ dos estudantes, $45,8 \%(n=60)$ situavam-se na faixa etária entre 23 e 26 anos, e $61,1 \%(n=80)$ eram estudante do internato. Entre os alunos entrevistados, $87,8 \%(n=115)$ não moravam em Mossoró antes de iniciarem a faculdade, e $45 \%(n=59)$ hoje moram sozinhos. Dos entrevistados, $56,5 \%(n=74)$ possuem excelente relação com os pais, $77,9 \%(n=102)$ não se consideram possuidores de insônia, e 58,8\% ( $n=57)$ afirmaram que apresentam um quadro de estresse diário (Tabela 1).

A prevalência do uso de SPA na vida foi de $81,7 \%$ ( $n=$ 107). As substâncias mais consumidas foram álcool $(78,6 \%$, $\mathrm{n}=103)$, ansiolíticos, sedativos e hipnóticos $(29,8 \%, \mathrm{n}=39)$, Cannabis $(22,9 \%, n=30)$ e tabaco $(21,4 \%, n=28)$. Em relação às outras substâncias pesquisadas, o consumo de inalantes, estimulantes do tipo anfetamina, alucinógenos, opiáceos e cocaína durante a vida foi, respectivamente, de $6,9 \%(n=9)$, $6,1 \%(n=8), 4,6 \%(n=6), 2,3(n=3)$ e $2,5 \%(n=2)$ (Tabela 2$)$.

Quando questionados sobre a substância mais frequentemente utilizada no dia a dia, 60,3\% dos estudantes ( $n=79$ ) mencionaram o álcool. Em seguida, obtiveram-se 
$18,3 \%$ ( $n=24$ ) com uso principal de ansiolíticos, sedativos e hipnóticos, e 1,5\% ( $n=2)$ e $0,8 \%(n=1)$, respectivamente, para Cannabis e tabaco. As demais SPA não foram escolhidas por nenhum discente. Outrossim, não houve diferença relevante na comparação desse consumo entre acadêmicos do primeiro ano e do internato (Tabela 3). Ademais, também não houve diferenças significativas quando esses dois grupos foram comparados quanto ao consumo individual de cada SPA, bem como no que concerne ao padrão de consumo.

Em relação ao padrão de consumo, $42,7 \%(n=56)$ dos estudantes fizeram uso de SPA nos últimos 30 dias; $19,8 \%$ ( $n=$ 26), nos últimos 12 meses; $20,6 \%(n=27)$, em qualquer período

Tabela 1. Descrição da amostra de estudantes de Medicina da Universidade do Estado do Rio Grande do Norte, $2021(n=131)$.

\begin{tabular}{llll}
\hline & Variável & n & $\%$ \\
\hline Sexo & & & \\
& Masculino & 79 & 60,3 \\
& Feminino & 52 & 39,7 \\
\hline
\end{tabular}

Idade

\begin{tabular}{ccc}
$18-22$ anos & 28 & 21,4 \\
$23-26$ anos & 60 & 45,8 \\
$27-30$ anos & 21 & 16 \\
$>30$ anos & 22 & 16,8 \\
\hline
\end{tabular}

Período do curso

\begin{tabular}{lll} 
Primeiro ano & 51 & 38,9 \\
Internato & 80 & 61,1 \\
\hline
\end{tabular}

Cursa Medicina em sua cidade de origem?

\begin{tabular}{ccc} 
Sim & 16 & 12,2 \\
Não & 115 & 87,8 \\
\hline
\end{tabular}

Relação com os pais

\begin{tabular}{ccc} 
Excelente & 74 & 56,5 \\
Boa & 49 & 37,4 \\
Regular & 7 & 5,3 \\
Ruim & 1 & 0,8 \\
\hline
\end{tabular}

Mora sozinho?

$$
\text { Sim }
$$

$59 \quad 45$

Não, moro com colegas

$27 \quad 20,6$

Não, moro com familiares

$45 \quad 34,4$

Tem insônia?

\begin{tabular}{lcc} 
Sim & 29 & 22,1 \\
Não & 102 & 77,9 \\
\hline
\end{tabular}

Apresenta um quadro de estresse?

\begin{tabular}{lll} 
Sim & 77 & 58,8 \\
Não & 54 & 41,2 \\
\hline
\end{tabular}

Fonte: Elaborada pelos autores. da vida; e 16,8\% ( $\mathrm{n}=22$ ) nunca utilizaram essas substâncias. Além disso, 58,8\% $(n=77)$ dos alunos acreditam que cursar Medicina é um fator precipitante para o consumo de SPA, sendo os principais motivos a carga horária extenuante $(49,6 \%, n=$ 65) e a privação do lazer $(43,5 \%, n=57)$. Quando questionados sobre o que a universidade poderia fazer para orientar os acadêmicos quanto ao uso das SPA, os participantes da pesquisa mencionaram: proporcionar assistência estudantil com equipe multiprofissional $(69,5 \%, n=91)$, abordar o tema nas aulas de farmacologia básica e aplicada $(62,6 \%, n=82)$, buscar fortalecer as relações interpessoais entre discentes, docentes e servidores $(62,6 \%, n=82)$, e organizar eventos temáticos $(53,4 \%, n=70)$.

Tabela 2. Descrição do uso na vida de substâncias psicoativas por estudantes de Medicina da Universidade do Estado do Rio Grande do Norte, 2021 ( $n=131$ ).

\begin{tabular}{ccc}
\hline Substância & n & $\%$ \\
\hline Tabaco (cigarros, charutos, cigarrilhas etc.) & & \\
Sim & 28 & 21,4 \\
Não & 103 & 78,6 \\
\hline
\end{tabular}

Álcool (cervejas, vinhos, licores, bebidas espirituosas, shots etc.)

\begin{tabular}{ccc} 
Sim & 103 & 78,6 \\
Não & 28 & 21,4 \\
\hline Cannabis (haxixe, erva, marijuana, pólen etc.) & & \\
Sim & 30 & 22,9 \\
Não & 101 & 77,1 \\
\hline Cocaína (coca, crack etc.) & & \\
Sim & 2 & 1,5 \\
Não & 129 & 98,5 \\
\hline
\end{tabular}

Estimulantes do tipo anfetamina (speed, anfetaminas, ecstasy etc.)

\begin{tabular}{ccc} 
Sim & 8 & 6,1 \\
Não & 123 & 93,1 \\
\hline Inalantes (cola, gasolina, óxido nitroso, solvente etc.) & \\
Sim & 9 & 6,9 \\
Não & 122 & 93,1 \\
\hline Ansiolíticos, sedativos e hipnóticos & \\
Sim & 39 & 29,8 \\
Não & 92 & 70,2 \\
\hline Alucinógenos (LSD, cogumelos, PCP, ketamina etc.) & \\
Sim & 6 & 4,6 \\
Não & 125 & 95,4 \\
\hline Sim & 3 & 2,3 \\
Não & 128 & 97,7 \\
\hline
\end{tabular}

Fonte: Elaborada pelos autores. 
Tabela 3. Substâncias psicoativas mais utilizadas pelos discentes de Medicina da Universidade do Estado do Rio Grande do Norte, 2021 (primeiro ano $=51$; internato $=80 ; n=131$ ).

\begin{tabular}{cccccccc}
\hline & & Tabaco & Álcool & Cannabis & $\begin{array}{c}\text { Ansiolíticos, sedativose } \\
\text { hipnóticos }\end{array}$ & Nenhum & Total \\
\hline Primeiro ano & $\%$ & $2,0 \%$ & $58,8 \%$ & $2,0 \%$ & $19,6 \%$ & $17,6 \%$ & $100 \%$ \\
Internato & $\%$ & $0,0 \%$ & $61,2 \%$ & $1,2 \%$ & $17,5 \%$ & $20,0 \%$ & $100 \%$ \\
\hline Total & $\%$ & $0,8 \%$ & $60,3 \%$ & $1,5 \%$ & $18,3 \%$ & $19,1 \%$ & $100 \%$ \\
\hline
\end{tabular}

Fonte: Elaborada pelos autores.

Tabela 4. Prevalência, no que concerne ao sexo, do consumo de tabaco e Cannabis entre os estudantes de Medicina da Universidade do Estado do Rio Grande do Norte, 2021 ( $n=131)$.

\begin{tabular}{cccccccccc}
\hline \multirow{2}{*}{ Variável } & \multicolumn{2}{c}{ Tabaco (Sim) } & \multicolumn{2}{c}{ Tabaco (Não) } & \multicolumn{2}{c}{ Cannabis (Sim) } & \multicolumn{2}{c}{ Cannabis (Não) } \\
\cline { 2 - 10 } & $\mathbf{n}$ & $\%$ & $\mathbf{n}$ & $\%$ & $\mathbf{n}$ & $\%$ & $\mathbf{n}$ & $\%$ \\
\hline Masculino & 11 & 13,9 & 68 & 86,1 & 13 & 16,5 & 66 & 83,5 \\
Feminino & 17 & 32,7 & 35 & 67,3 & 17 & 32,7 & 35 & 67,3 \\
\hline p valor & \multicolumn{3}{c}{0,019} & \multicolumn{3}{c}{0,05} \\
\hline
\end{tabular}

Fonte: Elaborada pelos autores.

Tabela 5. Associações entre o consumo de ansiolíticos, sedativos e hipnóticos e as variáveis investigadas nos alunos de Medicina da Universidade do Estado do Rio Grande do Norte, 2021 ( $n=131$ ).

\begin{tabular}{|c|c|c|c|c|c|}
\hline \multirow{2}{*}{$\begin{array}{c}\text { Variável } \\
\text { Tem insônia? }\end{array}$} & \multicolumn{2}{|c|}{ Ansiolíticos, sedativos, hipnóticos (Sim) } & \multicolumn{3}{|c|}{ Ansiolíticos, sedativos, hipnóticos (Não) } \\
\hline & $\mathrm{n}$ & $\%$ & $\mathrm{n}$ & $\%$ & p valor \\
\hline Sim & 14 & 48,3 & 15 & 51,7 & \multirow{2}{*}{0,025} \\
\hline Não & 25 & 24,5 & 77 & 75,5 & \\
\hline Considera-se dependente? & $\mathrm{n}$ & $\%$ & $\mathrm{n}$ & $\%$ & $\mathrm{p}$ valor \\
\hline Sim & 6 & 85,7 & 1 & 14,3 & \multirow{2}{*}{0,004} \\
\hline Não & 33 & 26,6 & 91 & 73,4 & \\
\hline $\begin{array}{l}\text { Acredita que cursar Medicina é um fator } \\
\text { precipitante para o uso dos psicoativos? }\end{array}$ & $\mathrm{n}$ & $\%$ & $\mathrm{n}$ & $\%$ & $\mathrm{p}$ valor \\
\hline Sim & 30 & 39 & 47 & 61 & \multirow{2}{*}{0,011} \\
\hline Não & 9 & 16,7 & 45 & 83,3 & \\
\hline
\end{tabular}

Fonte: Elaborada pelos autores.

O consumo de tabaco e Cannabis foi significativamente maior em mulheres em relação aos homens ( $p=0,019$ e $p=0,05$, respectivamente) (Tabela 4). Além disso, 48,4\% dos discentes com insônia, 85,7\% dos que relataram possuir dependência e 39\% dos que acreditam que cursar Medicina é fator precipitante de consumo de SPA fazem uso de ansiolíticos, sedativos e hipnóticos, $p=0,025, p=0,004$ e $p=0,01$, respectivamente (Tabela 5).

\section{DISCUSSÃO}

A fase de transição do ensino médio para a faculdade é repleta de dúvidas e incertezas. Na faculdade, inicia-se uma trajetória, em tempo integral e durante seis anos, que expõe o estudante a diversos fatores estressantes ${ }^{3}$. Essa experimentação acadêmica gera possibilidades vivenciais diferenciadas e perspectivas futurísticas profissionais, sendo também um período crítico por vulnerabilizar o estudante para iniciar a ingestão de SPA e prosseguir com ela'. Atualmente, o uso de SPA tem sido considerado um impasse preocupante, uma vez que predispõe a acidentes, comportamentos de risco, distúrbios do sono e até mesmo dependência, tanto física quanto psicológica ${ }^{13}$.

Os jovens representam a maior parcela daqueles que usam drogas e os que mais consomem tais substâncias em excesso, constatação que também preocupa, uma vez 
que constituem o grupo populacional mais vulnerável às consequências desse uso ${ }^{14}$. Um estudo com 567 estudantes da área da saúde realizado na Universidade Federal dos Vales do Jequitinhonha e Mucuri (UFVJM) mostrou prevalência de "uso na vida" de $88,4 \%{ }^{15}$. Nesta pesquisa, encontrou-se valor de $81,7 \%$. Elevados valores de consumo entre estudantes universitários do Brasil foram encontrados também em diversos outros estudos, os quais apontaram o uso de drogas por parte dos acadêmicos de Medicina com objetivos de melhorar a performance acadêmica, reduzir o estresse psicológico e compensar a falta de tempo e de lazer ${ }^{16,17}$.

Seguindo uma tendência mundial, em estudos realizados com acadêmicos de Medicina no Brasil, o álcool ainda é a SPA mais consumida, seguido do tabaco e da maconha ${ }^{18-21}$. Entretanto, as SPA apresentadoras de maior relevância no presente trabalho, no que tange à ingesta, foram álcool $(78,6 \%)$, ansiolíticos, sedativos e hipnóticos (29,8\%), Cannabis $(22,9 \%)$ e tabaco $(21,4 \%)$. No que tange ao padrão de temporalidade do consumo das SPA, observa-se uma concentração de ingesta nos últimos 30 dias (42,7\%). Essa realidade é, possivelmente, contextualizada no andamento do curso e em suas exigências e consequentes necessidades de válvulas de escape diversificadas por parte do alunado.

O álcool é a droga legal mais usada no Brasil, ingerida por quase $70 \%$ da população ${ }^{22}$. No presente estudo, a prevalência para uso de álcool na vida foi de $78,6 \%$, índice semelhante aos achados de Gomes et al. ${ }^{23}$, que constataram que $81,1 \%$ de discentes já haviam feito uso de álcool na vida. Vários fatores podem contribuir para o consumo de álcool pelos estudantes universitários. Possivelmente, o prevalecimento alcoólico dá-se em razão da legalização e da amplitude da aceitação social do composto $^{24}$. Além disso, tem-se o fato de que a universidade representa uma oportunidade de independência, estimulando uma maior socialização e exposição a ambientes de festas, locais onde o álcool é frequentemente encontrado ${ }^{25}$. Outrossim, no caso deste trabalho, $87,8 \%$ dos estudantes tiveram que mudar de cidade para cursar Medicina, e essa situação de afastamento da família pode desencadear sentimentos de solidão e depressão que também se correlacionam com o maior consumo de álcool ${ }^{23}$.

Quando se analisou a segunda SPA mais consumida, verificou-se o prevalecimento dos ansiolíticos, sedativos e hipnóticos, o que é discordante da literatura nacional que aponta resultados com predomínio do tabaco nessa posição. Entretanto, apesar de o tabaco ocupar a posição de quarto lugar neste trabalho, esta pesquisa mostrou uma prevalência de consumo de tabaco $(21,4 \%)$, concordante em relação a estudos semelhantes envolvendo estudantes de Medicina no Espírito Santo e em faculdades no Nordeste, que evidenciaram prevalências de uso de tabaco de $20,1 \%$, $22 \%$ e $24,3 \%{ }^{20,26,27}$. Nessa perspectiva, os dados mais recentes do ano de 2019, a partir da Pesquisa Nacional de Saúde (PNS) ${ }^{28}$, apontam o percentual total de adultos fumantes em 12,6\%. Essa diferença de valores pode ser explicada pelo fato de essa amostra de estudantes ser composta de uma população mais jovem que vivencia um ambiente competitivo e desafiador, que pode levar o aluno a uma sensação de frustração e cobrança diante do fracasso, e suscitar o consumo dessa e de outras drogas ilícitas ${ }^{29}$.

Outrossim, visualizou-se a manutenção do perfil de consumo ao longo dos períodos da graduação que foram pesquisados, cenário que se contrapõe a diversos estudos que relataram um relevante padrão de crescimento desse consumo ao decorrer do curso de Medicina ${ }^{3,11,16,20,23,30}$. Esse resultado divergente sinaliza um acesso da juventude universitária cada vez mais precoce às SPA, inclusive antes do início da vida universitária, o que justifica tal achado. Essa situação é ainda mais preocupante quando se raciocina que o lógico seria ocorrer um decréscimo do consumo de SPA à medida que se espera que os futuros médicos tenham consciência acerca dos prejuízos do uso dessas substâncias e, ainda, sejam um exemplo para os pacientes, sobretudo para aqueles que precisam de orientação quanto ao uso de drogas.

Outro resultado interessante avaliado foi o significativo maior consumo de tabaco e Cannabis por mulheres de 32,7\% para ambas as drogas ( $p=0,019$ e $p=0,05$, respectivamente). Esse achado é dissonante de outras bibliografias levantadas que estudaram jovens universitários brasileiros, as quais relataram consumo estatisticamente significante maior tanto de tabaco quanto de Cannabis por homens ${ }^{1,30-32}$. Uma hipótese levantada pelos pesquisadores e justificadora dessa descoberta discrepante reside na possibilidade de as mulheres participantes da pesquisa encontrarem no consumo de tabaco e Cannabis uma possibilidade de sanar suas demandas individuais e acadêmicas com compostos de menor apelação social, culminando em menor visibilidade e estereotipação da coletividade sobre a conduta da mulher. Vê-se que a associação foi ainda mais significante entre mulheres e tabaco, uma droga há décadas manifestadamente consumida em decréscimo social. Isso, conjuntamente com os outros achados do trabalho, sugere que não há uma padronização genérica já esperada do perfil de consumo de SPA entre graduandos de Medicina. Dessa forma, deve-se falar, então, em hábitos e comportamentos configurados em heterogeneidade, consoante a motivação e os contextos da ambiência acadêmica do alunado, bem como da disponibilidade locorregional de compostos para os discentes.

No estudo em questão, a prevalência de uso na vida de ansiolíticos, sedativos e hipnóticos correspondeu a 29,8\%, sendo a segunda SPA mais consumida. Contudo, tanto Lemos et al. ${ }^{11}$ 
quanto Petroianu et al. ${ }^{19}$ encontraram, respectivamente, $11,9 \%$ e $12 \%$ de uso na vida de ansiolíticos, sedativos e hipnóticos em pesquisas equivalentes com estudantes de Medicina. Segundo Brito etal. ${ }^{33}$ emestudo realizado com 870 estudantes de Medicina, conforme o avançar do curso, observam-se um aumento no consumo desse tipo de SPA e uma piora na qualidade do sono, consequências que poderão ser criadas se se considerarem o aumento das exigências acadêmicas e o esgotamento físico e mental. Destaca-se ainda, na presente pesquisa, que $48,4 \%$ dos discentes que manifestaram ter insônia, 85,7\% dos que relataram possuir dependência e $39 \%$ dos que acreditam que cursar Medicina é fator precipitante de consumo de SPA fazem uso de ansiolíticos, sedativos e hipnóticos ( $p=0,025, p=0,004$ e $p=0,01$, respectivamente). Essa incorporação medicamentosa significa potencialmente o manejo de problemas contextuais e estruturais pelos quais o discente de Medicina perpassa, e, por isso, ele sente a necessidade de tal usufruto ${ }^{29}$.

Diante dessa realidade existente, diversas hipóteses são formuladas para tentar explicar tão relevante quadro de consumo de SPA. A priori, esse grupo de indivíduos apresenta menor qualidade de vida quando confrontado ao restante da população no que diz respeito à saúde psicológica e às relações sociais $^{8}$. Em pesquisa realizada com acadêmicos de Medicina da UERN, Cardoso Filho et al. ${ }^{34}$ constataram, em uma amostra de 72 discentes, que $45 \%$ se sentiam sobrecarregados por atividades extracurriculares e como consequência dispunham de pouquíssimos momentos de lazer. No atual estudo, 58,8\% $(n=77)$ dos acadêmicos responderam que cursar Medicina é um fator precipitante para o consumo de SPA, sendo os principais motivos a carga horária extenuante $(n=65)$ e a privação de lazer $(n=57)$.

Portanto, salienta-se a importância de a universidade e suas estruturas administrativas tomarem ciência da realidade como intuito de possibilitara criação de mecanismos assistenciais para esses acadêmicos. O conhecimento do perfil discente relativo à temática é primordial para a construção de políticas e estratégias focadas na prevenção e no combate do uso dessas substâncias. O sistema público de saúde e as universidades têm a responsabilidade de conduzir pesquisas e intervir no consumo de estimulantes entre acadêmicos de Medicina ${ }^{35}$. Destacam-se, assim, estratégias de intervenções voltadas para o apoio pedagógico e psicológico. No arremate, faz-se necessário criar ações que visem orientar e prevenir o consumo de SPA, lembrando que a melhoria do ambiente universitário é essencial nesse contexto, tornando-se fundamental que seja provocada a reflexão do tema no meio acadêmico.

Com base nos resultados obtidos, sugere-se que o curso de Medicina é fator estimulante para o consumo de SPA. Para além do fator tipo de graduação, os contextos sociodemográficos, como a idade jovem e o uso e o apelo social que fomenta o consumo diverso de psicoativos, devem ser considerados porque também predispõe o uso dessas substâncias. É preciso destacar que tais discentes serão futuros médicos, responsáveis pelo cuidado e pela promoção de saúde na comunidade. Assim, este estudo evidencia um resultado preocupante que ecoa na saúde pública, o qual é essencial que seja compreendido pelos representantes das universidades.

Destarte, convém destacar que este estudo apresenta como limitação o fato de ter sido realizado por questionário online, já que não foi possível aplica-lo presencialmente em razão da pandemia da coronavírus disease 2019 (Covid-19). Ademais, por conta da complexidade e dos múltiplos fatores acerca do tema, faz-se imperativo que estudos a posteriori ampliem as populações estudantis médicas estudadas, as quais se revelam heterogêneas e não padronizadas em termos de perfil de consumo dessas substâncias.

\section{CONCLUSÃO}

Constata-se elevado uso na vida de SPA entre os estudantes de Medicina da UERN, perfil que se mostrou semelhante entre os semestres pesquisados, ao longo da evolução do curso. É exibido um perfil majoritariamente alcoólico, seguido de medicamentos ansiolíticos, sedativos e hipnóticos, Cannabis e tabaco, com prevalecimento temporal chamativo nos últimos 30 dias, indicando utilização constante e perene. A segunda posição de consumo das SPA mencionadas, em contraponto à literatura, sinaliza um cenário preocupante de tentativa de medicalização discente diante dos contextos vivenciados.

A pesquisa averiguou uma diferença significativamente maior do consumo de Cannabis e tabaco por mulheres em comparação com os homens, achado dissonante das literaturas utilizadas para o embasamento do trabalho. Além disso, demonstrou relação relevante quanto à prevalência de insônia e à dependência entre os estudantes que utilizam ansiolíticos, sedativos e hipnóticos, e o fato de acreditarem que cursar Medicina é um fator precipitante para o uso de SPA.

\section{AGRADECIMENTOS}

Aos estudantes da FACS que responderam ao questionário e ao Programa Institucional de Bolsas de Iniciação Científica (PIBIC) da UERN.

\section{CONTRIBUIÇÃO DOS AUTORES}

Sabrina Mércia dos Santos Siebra e Tassio Danilo Rego de Queiroz participaram da concepção do artigo, da coleta e análise dos dados, e da escrita e revisão final do manuscrito. Eudes Euler de Souza Lucena participou da análise dos dados 
e da revisão final do manuscrito. Allyssandra Maria Lima Rodrigues Maia e Ubirajara Caldas Leonardo Nogueira Junior participaram da revisão final do manuscrito. Álvaro Marcos Pereira Lima participou da concepção do artigo, da análise dos dados e da revisão final do manuscrito.

\section{CONFLITO DE INTERESSES}

Declaramos não haver conflito de interesses.

\section{FINANCIAMENTO}

Os alunos participaram do PIBIC da UERN, edição 2020/2021 ID: 2444.

\section{REFERÊNCIAS}

1. Fernandes TF, Monteiro BMM, Silva JBM, de Oliveira KM, Viana NAO, da Gama GAP, et al. Uso de substâncias psicoativas entre universitários brasileiros: perfil epidemiológico, contextos de uso e limitações metodológicas dos estudos. Cad Saude Colet. 2017;25(4):498-507.

2. Coutinho C, Toledo L, Bastos FI. Epidemiologia do uso de substâncias psicoativas no Brasil. Rio de Janeiro: Fundação Osvaldo Cruz; 2019. p. 1-27 [acesso em 10 ago 2021]. Disponível em: https://saudeamanha.fiocruz.br/ wp.content/uploads/2020/03/PJSSaudeAmanha_Texto0039_v02.pdf.

3. de Andrade JBC, Sampaio JJC, de Farias LM, Melo LP, de Sousa DP, de Mendonça ALB, et al. Contexto de formação e sofrimento psíquico de estudantes de medicina. Rev Bras Educ Med. 2014;38(2):231-42.

4. Pires AMFS, Gusmão WDP, de Carvalho LWT, do Amaral MMLS. Qualidade de vida de acadêmicos de Medicina: há mudanças durante a graduação? Rev Bras Educ Med. 2020;44(4):1-7.

5. Silva RC, Pereira AA, Moura EP. Qualidade de vida e transtornos mentais menores dos estudantes de Medicina do Centro Universitário de Caratinga (Unec) - Minas Gerais. Rev Bras Educ Med. 2020;44(2):1-7.

6. Loureiro EMF, Mcintyre T, Cardoso RM, Ferreira MA. A relação entre o stress e os estilos de vida nos estudantes de medicina da Faculdade de Medicina do Porto. Rev Bras Educ Med. 2008;21(3):209-14.

7. Pupulim AF, Sarris AB, Fernandes LGR, Nakamura MC, Camargo TV, Paula JB. Mecanismos de dependência química no tabagismo: revisão da literatura. Rev Méd UFPR. 2015;2(2):1-74.

8. Miranda IMM, Tavares HHF, da Silva HRS, Braga MS, Santos RO, Guerra HS. Qualidade de vida e graduação em Medicina. Rev Bras Educ Med. 2020;44(3):1-8.

9. Machado CS, de Moura TM, de Almeida RJ. Estudantes de Medicina e as drogas: evidências de um grave problema. Rev Bras Educ Med. 2015;39(1):159-67.

10. Universidade do Estado do Rio Grande do Norte. Projeto Pedagógico do Curso de Medicina da Faculdade de Ciências da Saúde. Mossoró: Uern; 2014 [acesso em 20 ago 2020]. Disponível em: https://www.uern.br/ controledepaginas/documentos-legislacao-ensino/arquivos/0065ad_ referendum_consepe_6_2014_aprova_o_ppc_de_medicina_do_ campus_central_anexos.pdf.

11. Lemos KM, Neves NMBC, Kuwano AY, Tedesqui G, Bitencourt AGV, Neves FBCS, et al. Uso de substâncias psicoativas entre estudantes de Medicina de Salvador (BA). Rev Psiquiatr Clín. 2007;34(3):118-24.

12. Smart RG, Hughes PH, Johnson LD. A methodology for student drug-use surveys. Geneva: World Health Organization; 1980. v. 50, p. 7-55.

13. Conceição LS, Batista CB, Dâmaso JGB, Pereira BS, Carniele RC, Pereira GS. Saúde mental dos estudantes de medicina brasileiros: uma revisão sistemática da literatura. Avaliação (Campinas). 2019;24:785-802.
14. Perini GB, Gonçalves LSSP, Svoboda NK, Monteiro LB, Prochmann IR, Muniz JO. Relatório Mundial sobre Drogas 2020: breves considerações da coordenação do Comitê do MPPR de Enfrentamento às Drogas. Curitiba: Ministério Público do Estado do Paraná; 2020. p. 1-13 [acesso em 10 jun 2021]. Disponível em: https://site.mppr.mp.br/arquivos/File/Relatorio_ Mundial_Drogas.pdf.

15. Santos DDM, Guimarães MM, Bodevan EC, Rocha RL, Pinheiro MLP. Use of psychoactive substances among university students. SMAD, Rev Eletrônica Saúde Mental Álcool Drog. 2019;15(3):1-9.

16. Paduani GF, Barbosa GA, de Morais JCR, Pereira JCP, Almeida MF, Prado $M M$, et al. Consumo de álcool e fumo entre os estudantes da Faculdade de Medicina da Universidade Federal de Uberlândia. Rev Bras Educ Med. 2008;32(1):66-74.

17. de Oliveira LG, Barroso LP, Wagner GA, Ponce JC, Malbergier A, Stempliuk VA, et al. Consumo de drogas entre estudantes de medicina em São Paulo: influências de gênero e ano letivo. Rev Bras Psiquiatr. 2009;31(3):227-39.

18. Antoniassi Júnior G, Gaya CM. Implicações do uso de álcool, tabaco e outras drogas na vida do universitário. Rev Bras Promoç Saúde. 2015;28(1):67-74.

19. Petroianu A, dos Reis DCF, Cunha BDS, de Souza DM. Prevalência do consumo de álcool, tabaco e entorpecentes por estudantes de medicina da Universidade Federal de Minas Gerais. Rev Assoc Med Bras. 2010;56(5):568-71.

20. Miranda CC, Azevedo GZ, Moreira BR, Pesca JPM, Destefani BP, Rizzi LM, et al. Análise do consumo de substâncias psicoativas por estudantes de medicina de uma Faculdade do Espírito Santo, Brasil. Arq Méd Hosp Fac Ciênc Méd Santa Casa São Paulo. 2020;65(1):1-8.

21. Tockus D, Gonçalves PS. Detecção do uso de drogas de abuso por estudantes de medicina de uma universidade privada. J Bras Psiquiatr. 2008;57(3):184-7.

22. Galduróz JCF, Noto AR, Nappo SA, Carlini EA. Uso de drogas psicotrópicas no Brasil: pesquisa domiciliar envolvendo as 107 maiores cidades do país - 2001. Rev Lat Am Enfermagem. 2005;13:888-95 [acesso em 10 ago 2020]. Disponível em: http://www.ncbi.nlm.nih.gov/pubmed/16400456.

23. Gomes LS, Barroso CRD, Silvestre VA, Baylão ACDP, Garcia SCM, Pacheco SJB. Consumo de álcool entre estudantes de medicina do Sul Fluminense - RJ. Rev Med (São Paulo). 2018;97(3):260-6.

24. Rocha LA, Lopes ACFMM, Martelli DRB, Lima VB, Martelli-Júnior H. Consumo de álcool entre estudantes de faculdades de Medicina de Minas Gerais, Brasil. Rev Bras Educ Med. 2011;35(3):369-75.

25. Mendonça AKRH, de Jesus CVF, Lima SO. Fatores associados ao consumo alcoólico de risco entre universitários da área da saúde. Rev Bras Educ Med. 2018;42(1):207-15.

26. Pereira DS, De Souza RS, Buaiz V, De Siqueira MM. Uso de substâncias psicoativas entre universitários de medicina da Universidade Federal do Espírito Santo. J Bras Psiquiatr. 2008;57(3):188-95.

27. Pinheiro MA, Torres LF, Bezerra MS, Cavalcante RC, Alencar RD, Donato $A C$, et al. Prevalência e fatores associados ao consumo de álcool e tabaco entre estudantes de Medicina no Nordeste do Brasil. Rev Bras Educ Med. 2017;41(2):231-9.

28. Pesquisa Nacional de Saúde. Dados e números da prevalência do tabagismo. Instituto Nacional de Câncer; 2019 [acesso em 10 ago 2020]. Disponível em: https://www.inca.gov.br/observatorio-da-politica-nacional-de-controledo-tabaco/dados-e-numeros-prevalencia-tabagismo\#: :text=Os dados mais recentes do,fumantes em 12\%2C6 \%25.

29. Vaz ALL, Gléria VO, Bastos CFC, Sousa IF, Silva AMTC, Almeida RJ. Fatores associados aos níveis de fadiga e sonolência excessiva diurna em estudantes do internato de um curso de Medicina. Rev Bras Educ Med. 2020;44(1):1-9.

30. Rodrigues Júnior GA, Queiroz FO, Lima HDN, Nóbrega LS, Oliveira IRN. Fatores associados ao uso de substâncias psicoativas em estudantes de uma universidade pública do sul do Maranhão. Rev Med (São Paulo). 2020;99(3):220-9.

31. Ferraz L, Rebelatto SL, Schneider GC, Anzolin V. O uso de álcool e tabaco entre acadêmicos de uma universidade do sul do Brasil. Rev Bras Promoç Saúde. 2017;30(1):79-85. 
32. Colares V, Da Franca C, Gonzalez E. Condutas de saúde entre universitários: diferenças entre gêneros. Cad Saude Publica. 2009;25(3):521-8.

33. Brito JR, Silva PR. Uma análise sobre o consumo de ansiolíticos e antidepressivos entre estudantes de Medicina [trabalho de conclusão de curso]. Goiânia: Pontifícia Universidade Católica de Goiás; 2021 [acesso em 15 jun 2021]. Disponível em: https://repositorio.pucgoias. edu.br/jspui/bitstream/123456789/2092/1/UMA\%20AN\%C3\%81LISE\%20 SOBRE\%200\%20CONSUMO\%20DE\%20ANSIOL\%C3\%8DTICOS\%20 E\%20ANTIDEPRESSIVOS\%20ENTRE\%20ESTUDANTES\%20DE\%20 MEDICINA\%20\%28corrigido\%29.pdf.
34. Cardoso Filho FAB, Magalhães JF, Silva KML, Pereira ISSD. Perfil do estudante de Medicina da Universidade do Estado do Rio Grande do Norte (UERN), 2013. Rev Bras Educ Med. 2015;39(1):32-40.

35. Urrego MA, Orozco LA, Montoya LB, Soto LB, Carmona VD, Castrillón JJC, et al. Consumo de anfetaminas, para mejorar rendimiento académico, en estudiantes de la Universidad de Manizales, 2008. Arch Med. 2009;9(1):43-57. 